\title{
The relationship between dreams and subsequent morning mood using self-reports and text analysis
}

\author{
Remington Mallett ${ }^{1}$ \\ Claudia Picard-Deland ${ }^{2}$ \\ Wilfred Pigeon ${ }^{3}$ \\ Madeline Wary ${ }^{3}$ \\ Alam Grewal ${ }^{3}$ \\ Mark Blagrove ${ }^{4}$ \\ Michelle Carr ${ }^{3,4 *}$
}

${ }^{1}$ Department of Psychology, University of Texas at Austin, USA

${ }^{2}$ Department of Neuroscience, Université de Montréal, Canada

${ }^{3}$ Sleep \& Neurophysiology Research Laboratory, University of Rochester Medical Center, USA

${ }^{4}$ Department of Psychology, Swansea University, UK

*corresponding author: michelle_carr@urmc.rochester.edu

\begin{abstract}
While material from waking life is often represented in dreams, it is less clear whether and how dreams impact waking life. Here, we assessed whether dream mood and content from home diaries predict subsequent waking mood using both subjective self-reports and an objective automated word detection approach. Subjective ratings of dream and morning mood were highly correlated within participants for both negative and positive valence, suggesting that dream mood persists into waking. Text analyses revealed similar relationships between affect words in dreams and morning mood. Moreover, dreams referencing death or the body were related to worse morning mood, as was first-person singular pronoun usage (e.g., "I"). Dreams referencing leisure or ingestion, or including first-person plural pronouns (e.g., "we"), were related to better morning mood. Together, these results suggest that subjective experiences during sleep, while often overlooked, may be an important contributor to waking mood.
\end{abstract}

\section{Keywords}

sleep, dreaming, emotion regulation, LIWC

\section{Acknowledgements}

The authors would like to thank Ashwini Ashokkumar and the rest of the Pennebaker Language Lab for helpful discussion. 
DREAMS AND MORNING MOOD

\section{Declarations}

\section{Funding}

This work was funded by the Mind \& Life Europe Francisco J. Varela Award (MC) and the National Institute of General Medical Sciences grant: K12 GM106997 (MC).

\section{Conflicts of interest/Competing interests}

The authors declare no potential conflict of interest.

\section{Availability of data and material}

Data and materials are available in an OSF repository: https://osf.io/2xy5p/

\section{Code availability}

Analysis code is available in an OSF repository: https://osf.io/2xy5p/

\section{Ethics approval}

Ethical approval was granted by the Swansea University Department of Psychology Research Ethics Committee.

\section{Consent to participate}

Informed consent was obtained from all participants included in the study.

\section{Consent for publication}

N/A 
DREAMS AND MORNING MOOD

\section{Introduction}

While many studies have emphasized a strong bidirectional relationship between sleep and mood (Vandekerckhove \& Cluydts, 2010), relatively few have addressed how subjective experiences during sleep influence subsequent waking affect. When retrospectively surveyed about the perception of how dreams influence their waking life, nearly $40 \%$ of participants report that their dreams impact their waking mood more than once a month (Schredl, 2000, 2009). In a diary study where participants reported the perceived influence of dreams on waking mood daily, both positive and negative dream moods influenced daily mood, especially when intense (Schredl \& Reinhard, 2010). This appears to be true for a variety of emotions, such as exhilaration and contempt (Yu, 2007), as well as cognitive features of dreams such as mindfulness and self-reflection (Lee \& Kuiken, 2015). On mornings after nightmares, participants report higher anxiety, sadness, and even physical pain (Köthe \& Pietrowsky, 2001), and having frequent nightmares is associated with higher waking distress and lower well-being (Blagrove et al., 2004).

Previous work has primarily relied on self-report measures of dream emotion to assess relationships with morning mood. Text analytic approaches can also be applied to dream reports to extract more objective affect ratings and other content scores (Bulkeley \& Graves, 2018; Hawkins II \& Boyd, 2017). In the current study, we combined these approaches to investigate how dream affect and other aspects of dream content influence morning mood. Towards this aim of replication and extension, we quantified similarities and differences between emotion in sleep and wake, and explored different aspects of dream content that influence morning mood.

\section{Methods}

\section{Participants}

Participants were recruited through the use of a university-wide email advertisement. In addition to general recruitment, we targeted individuals who recall weekly bad dreams (defined as a mild negative dream) or nightmares (defined as an intense negative dream that wakes you suddenly). Potential candidates completed a screening questionnaire in which they selfreported their typical recall frequencies per month of three categories of dreams: neutral or positive dreams, bad dreams, and nightmares, using a 6-point scale (1 = never, 2 = once per month, $3=$ two to three times per month, $4=$ once per week, $5=$ two to four times per week, or $6=$ most days). Candidates were also screened and excluded for severe depressive or posttraumatic stress symptoms. More detail is available in the parent study, which assessed brain activity (see Carr, Summers, et al., 2020). Fifty-five eligible participants were enrolled in the study and signed Informed Consent. The final sample consisted of 54 participants (1 
DREAMS AND MORNING MOOD

40 excluded for failure to complete dream diaries). The mean age of the sample was 23.9 years

$41 \quad(S D=4.2 ; 35$ females, 19 males). Ethics were approved by the Swansea University

42 Department of Psychology Research Ethics Committee.

43

\section{Procedure}

Each morning participants filled in a brief dream diary via PsyToolkit (Stoet, 2017) that contained questions about their sleep and dream quality of the previous night. Participants completed up to 8 weeks of dream diaries during the study. Participants were first asked about sleep parameters including time to bed, minutes to fall asleep, minutes awake during the night, and time of waking. These questions were followed by a dream report and six scaled Likert questions (1-9 scale, with 1 being a very low level, 5 being a moderate level, and 9 being an intense level of the designated attribute; additional no recall option) regarding the intensity of negative and positive dream emotions, the amount of negative and positive dream body sensations, and the extent to which one was distressed or in a good mood upon awakening from the dream, all with respect to their most recent dream.

\section{Analysis}

Preprocessing and report exclusion. Dream reports were stripped of all non-dream content via manual inspection. Two manual examiners were directed to remove all contextual material, commentary, instances of waking explanations, explicit references to the dream (e.g., "In the dream..."), and hesitations, stutters, or word repetitions. To remove morning reports with little to no dream content, reports with fewer than 30 words were removed $(68 \%$ of entries). Because we were primarily interested in dream and wake emotion, all reports without any recall of all four of dream positive emotion, dream negative emotion, wake positive emotion, and wake negative emotion were removed (leaving $92 \%$ of reports that survived word restriction). After such exclusions, we removed any participants without 2 or more reports left $(N=4)$. The final dataset consisted of 671 dream reports from 46 participants. After exclusions, participants reported on average 14.6 dream reports $(S D=9.4)$, with a median of 12 and a range from 2 to 38 reports.

Correlation and emotion difference analysis. To quantify the relationship between selfreported dream emotions and wake emotions, we correlated dream and wake emotion separately for positive and negative valence. We used a repeated measures correlation, which takes advantage of sample dependence within participants and attempts to find a regression slope that is common across all participants (Bakdash \& Marusich, 2017). To quantify change in emotion from dreaming to waking, we subtracted reported dream emotion intensity from reported wake emotion intensity, separately for each valence. Thus, a positive value results from increased emotion intensity from dreaming to waking, and a negative value results from decreased emotion intensity from dreaming to waking. Change in emotion intensity was averaged within each participant across mornings and compared against zero using a twotailed one-sample $t$-test. A paired $t$-test was used to compare the change in emotion intensity 
80 between positive and negative valence. These statistics were implemented using the Pingouin 81 statistical Python package (Vallat, 2018).

$82 \quad$ LIWC and regression analysis. Dream reports were submitted to Linguistic Inquiry and 83 Word Count 2015 (LIWC), a text analysis software that takes a dictionary-based approach by returning word frequencies of a variety of validated categories (Pennebaker et al., 2015). Categorical word frequencies offered by LIWC include common linguistic features (e.g., articles and pronouns), psychological processes (e.g., positive emotion and certainty words), content words (e.g., family and body words), and punctuation (e.g., question marks and semicolons). We selected a subset of LIWC categories related to common aspects of dreaming and emotion in order to evaluate the relationship between dream content and waking emotion. LIWC categories were binned into superordinate groups for visualization and interpretation purposes (affect, personal concerns, characters, drives, and sensory). The affect group consisted of LIWC categories anxiety, anger, sadness, and positive emotion (abbreviated pos emo). The personal concerns group consisted of LIWC categories death, religion, home, work, money, and leisure. The characters group consisted of male, female, friend, family, first-person singular pronouns (abbreviated $i$ ), and first-person plural pronouns (abbreviated we). The drives group consisted of risk, power, reward, affiliation, and achievement (abbreviated achieve). The sensory group consisted of body, feel, hear, health, see, sexual, and ingestion. See Pennebaker et al. (2015) for example words from each category.

For each relevant LIWC category, word frequencies were derived for each dream report and submitted to a linear mixed effects model with waking negative emotion as the outcome variable, word frequency as predictor, and participant ID as random effect. An additional analysis was run including sleep efficiency as an additional predictor, where sleep efficiency was calculated as total time asleep (time in bed minus sleep latency and WASO) divided by total time in bed (risetime minus bedtime), multiplied by 100. Though both negative and positive waking emotion were reported each morning, we chose only one as the outcome predictor because the two responses were correlated $(r=-.35, p<.001)$. We chose negative over positive emotion because of its clinical relevance, and because negative waking mood responses had wider response variation in the current dataset. Results were qualitatively similar when using positive waking mood as the outcome variable. All predictors and the outcome variable were $z$-scored to allow for the reporting of standardized regression coefficients. Reported $p$-values were corrected for multiple comparisons across all word categories (28 tests) by adjusting for the false discovery rate (FDR) using the BenjaminiHochberg step-up procedure. This analysis was performed using the statsmodels statistical Python package.

\section{Results}


DREAMS AND MORNING MOOD

Across our final sample $(N=46)$, average self-reported dream recall frequencies on the 6-point scale were $4.4(S D=1.2)$ for neutral or positive dreams (i.e., between one and four neutral/positive dreams per week), $3.6(S D=1.5)$ for bad dreams (i.e., between two and four bad dreams per month), and $2.4(S D=1.3)$ for nightmares (i.e., between one and three nightmares per month). For all further descriptive summary statistics, including average number of dream reports, dream word counts, dream and wake emotion ratings, sleep efficiency and LIWC variables, see Supplementary Information.

Across participants, dream and waking mood were highly correlated for both negative $(r$ $=.65, p<.001)$ and positive valence $(r=.55, p<.001$; Figure 1A). For negative valence, the change in emotion intensity from dream to wake was below zero $\left(t_{45}=8.7, p<.001\right.$, Cohen's $d$ $=1.29$ ), suggesting a dissipation of negative emotion from dream to wake (Figure 1B). There was no change in emotion intensity for positive emotion ( $t_{45}=1.5, p=.134$, Cohen's $d=.22$ ). The change in emotional intensity was lower for negative valence than for positive valence $\left(t_{45}\right.$ $=6.1, p<.001$, Cohen's $d=1.53$ ).

A)
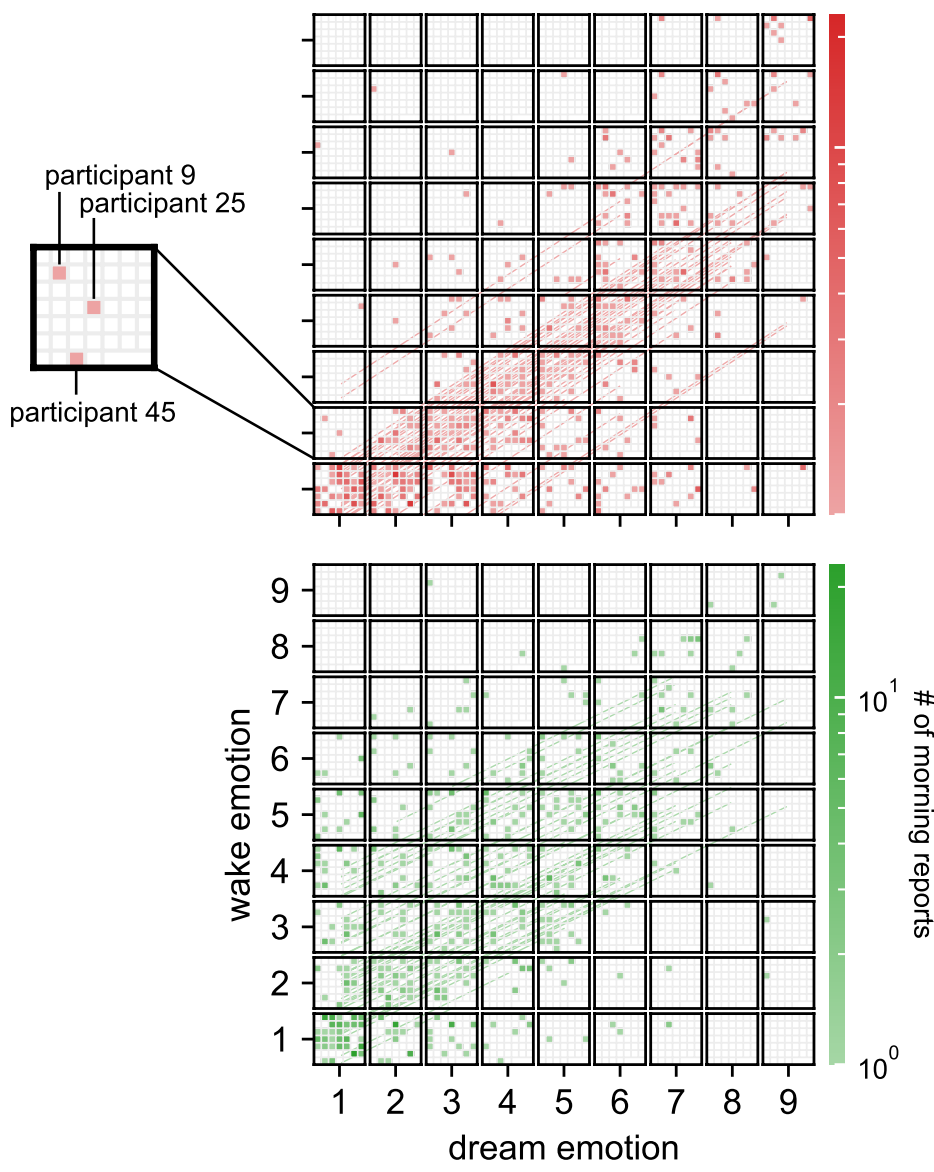

B)

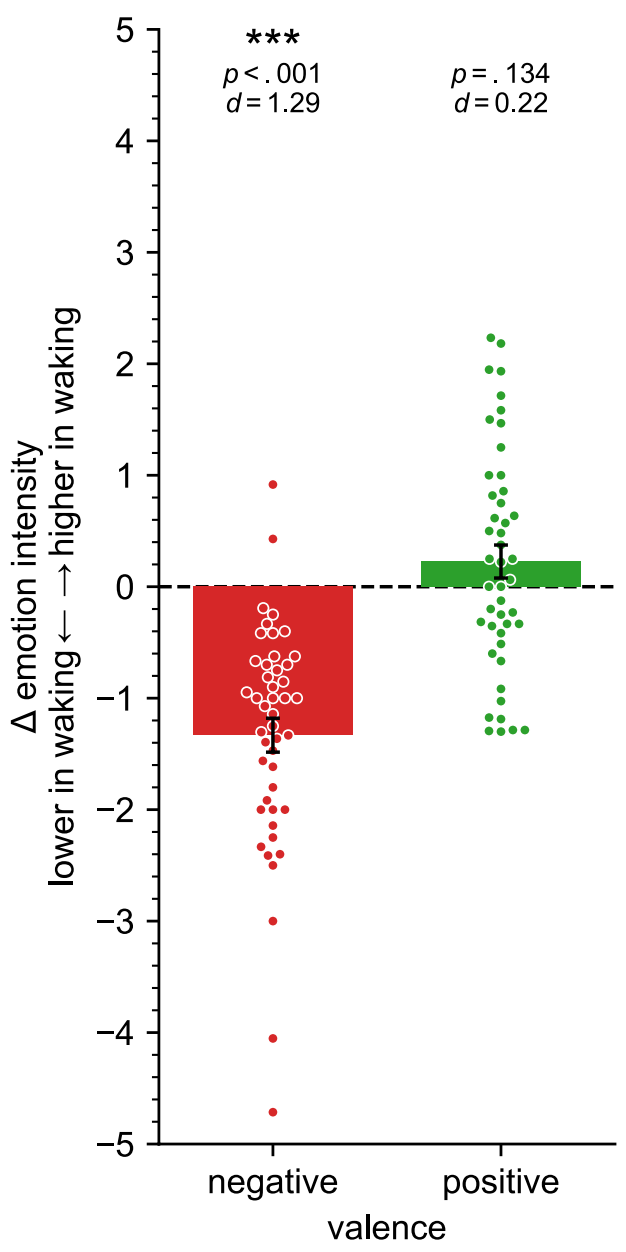




\section{DREAMS AND MORNING MOOD}

ratings were highly correlated across dreaming and waking. As a whole, both graphs illustrate results of the repeated measures correlation analysis with regression lines drawn for each participant. Within each graph, constituent grids at each Likert pairing reveal heatmaps, in which each individual square represents a single participant, and the shading of each square indicates the number of reports that participant contributes to the given Likert pairing. Ordering of participants is consistent across heatmaps. (B) Negative emotion, on average, decreased in intensity after awakening. Positive emotion was unchanged between dreaming and waking, though trended as increasing in waking. Each datapoint represents the average difference between reported waking and dreaming emotion intensity for a single participant.

Language predictors are presented according to our custom superordinate groups, which are largely consistent with standard LIWC categories (Figure 2). Unsurprisingly, affect/emotion word categories had the most predictive power on morning mood, with anxiety words predicting increased negative morning mood $\left(B=.14, p_{\mathrm{fdr}}<.001\right)$ and positive emotion words predicting decreased negative morning $\operatorname{mood}\left(\beta=-.16, p_{\mathrm{fdr}}<.001\right)$, while other affect words were not predictive (anger and sadness, $p_{\mathrm{fdr}}$ 's $>.25$ ). Word categories regarding personal concerns were predictive of morning mood. In particular, death words were predictive of increased negative morning $\operatorname{mood}\left(B=.09, p_{\mathrm{fdr}}=.030\right)$, and leisure words were predictive of decreased negative morning mood $\left(\beta=-.10, p_{\mathrm{fdr}}=.012\right)$. Money words trended in the direction of predicting less negative morning mood $\left(\beta=-.07, p_{\mathrm{fdr}}=.078\right)$ and others were not predictive $\left(p_{\mathrm{fdr}}\right.$ 's $\left.>.62\right)$. Character word categories were at times predictive of morning mood, primarily from pronoun usage. First-person singular pronouns were highly predictive of increased negative morning mood $(\beta=.12, p=.005)$, while first-person plural pronouns were predictive of less negative morning mood $(\beta=-.09, p=.030)$. Beyond pronouns, male words trended as predictive of more negative morning $\operatorname{mood}\left(\beta=.07, p_{\mathrm{fdr}}=.078\right)$ and no other categories were predictive $\left(p_{\mathrm{fdr}}\right.$ 's $>$.62). Word categories regarding drives were not strongly predictive of morning mood, with only risk words trending $\left(\beta=.07, p_{\mathrm{fdr}}=.125\right)$ as predictive of increased negative morning mood (all other $p_{\mathrm{fdr}}$ 's $>.14$ ). Of sensory word categories, words relating to the body were predictive of increased negative morning mood $\left(\beta=.09, p_{\mathrm{fdr}}=.030\right)$, words relating to ingestion were predictive of decreased negative morning $\operatorname{mood}\left(\beta=-.09, p_{\mathrm{fdr}}=\right.$ .030 ), and other sensory categories were not predictive ( $p_{\mathrm{fdr}}$ 's $\left.>.31\right)$. Results were qualitatively similar when controlling for sleep efficiency, which itself was not a significant predictor of morning mood in any of the models (see Supplementary Information). 
DREAMS AND MORNING MOOD

172
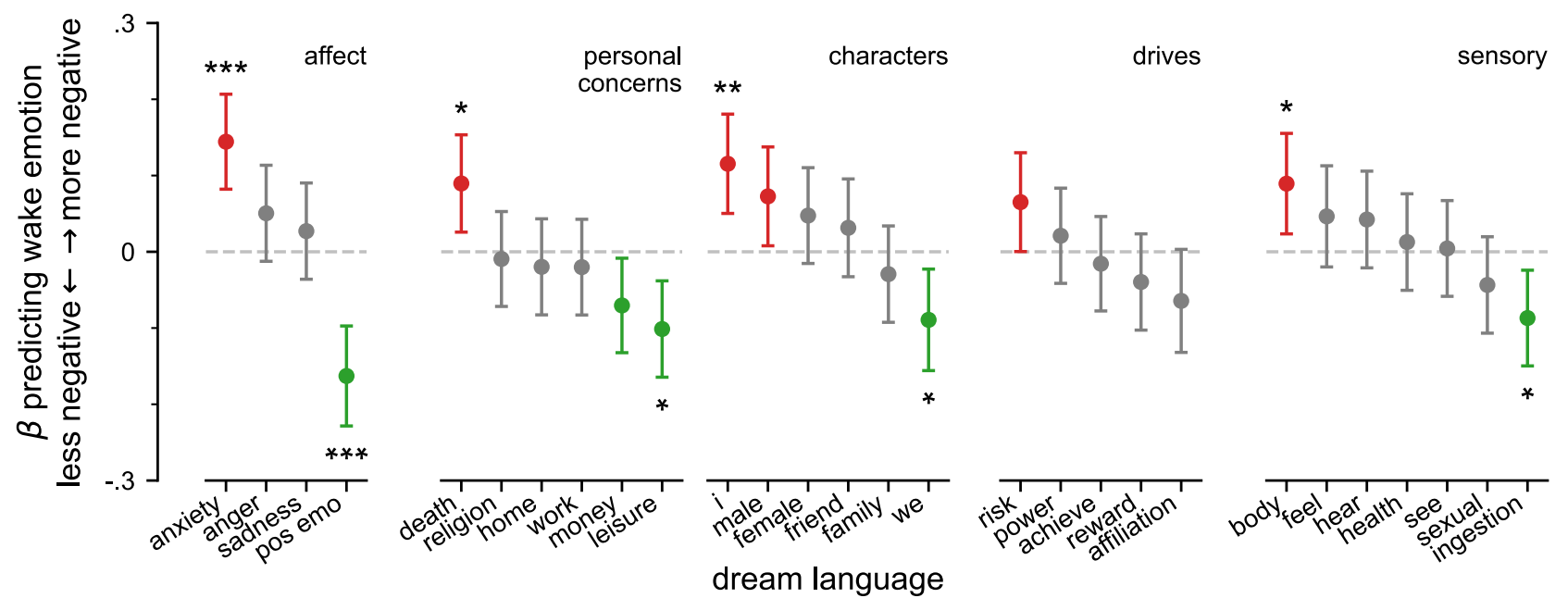

Figure 2. Automated measures of dream content predict negative morning mood. Anxiety, death, body, and " $i$ " words were strong predictors of high negative morning emotion. Positive emotion, leisure, ingestion, and "we" words were strong predictors of low negative morning emotion. Error bars represent 95\% confidence intervals. Points are colored if significant at uncorrected level of $p<.05$ (red for positive betas predicting increased negative emotion, green for negative betas predicting decreased negative emotion). FDR-corrected $p$-values indicated with asterisks at levels of ${ }^{*} p_{\mathrm{fdr}}<.05,{ }^{* *} p_{\mathrm{fdr}}<.01$, ${ }^{* * *} p_{\mathrm{fdr}}<.001$.

\section{Discussion}

Overall, results suggest that dreaming and subsequent waking mood are tightly bound. First, self-reported dream emotion was strongly predictive of waking mood, though on average negative dream emotion dissipated prior to or during waking. Second, text analysis applied to dream reports verified the close relationship between dreaming and morning mood, and revealed other content categories related to morning mood. Dreams of anxiety, death, the body, and first-person singular references related to more negative morning mood. Dreams of positive emotion, leisure, ingestion, and plural first-person references related to less negative morning mood. These effects occurred despite controlling for sleep quality (see also Barnes et al., 2020). Nevertheless, these findings are still limited only to dreams which can be remembered upon waking.

The spillover of dreaming into waking mood is reminiscent of sleep inertia, where impaired cognitive performance persists for a short period upon awakening. Dream emotion may lead to a similar type of "dream inertia" that persists on waking and dissipates over time. Our insight that, when morning mood does diverge from dream emotion it is typically less negative, is consistent with a putative function of REM sleep in processing emotional events 
DREAMS AND MORNING MOOD

200 and reducing the emotional tone of memories (Walker \& van der Helm, 2009). Future work 201 collecting mood reports across the day, and for several days, would better elucidate both the 202 directionality and time-course of this potential dream inertia.

203 Previous research on nightmares is also broadly consistent with the content that we 204 found predictive of negative morning mood. Nightmares contain low rates of positive, and high 205 rates of negative, emotion words (Bulkeley \& Graves, 2018). Death is a common theme of 206 nightmares (Bulkeley \& Graves, 2018; Fireman et al., 2014; Schredl \& Göritz, 2018) and 207 body/somatosensory content is frequent in dysphoric dreaming, including threats of physical 208 harm, falling, and paralysis (Robert \& Zadra, 2014; Schredl \& Göritz, 2018). Most aggressors 209 in nightmares are, incidentally, male perpetrators (Schredl \& Göritz, 2018). Post-trauma 210 nightmares, compared with typical dreams, have reduced leisure word counts and increased 211 risk words, as well as higher rates of singular first-person pronouns (Paquet et al., 2020). 212 Notably, "finding money" and "eating delicious foods" are both positive typical dream themes 213 (Nielsen et al., 2003). All of these patterns are consistent with word categories that we found 214 predictive of morning mood, though it is important to consider the limitation that our sample 215 was biased towards those that suffer from dysphoric dreaming.

Together, these findings begin to disentangle relationships between subjective sleep experience and waking mood and reveal specific affect and content categories in dream reports relevant to this relationship. With the ability to engineer and manipulate dream content on the horizon (Carr, Haar, et al., 2020), the identification of specific dream content that boosts waking mood could inform interventions that aim to improve well-being or reduce waking distress, such as lucid dreaming (Konkoly \& Burke, 2019; Stocks et al., 2020). In sum, while oft observed that daily experience influences our nightly dreams, the present results suggest that dreams recalled upon awakening also influence subjective experience the following day. 
DREAMS AND MORNING MOOD

\section{References}

Bakdash, J. Z., \& Marusich, L. R. (2017). Repeated Measures Correlation. Frontiers in Psychology, 8. https://doi.org/10.3389/fpsyg.2017.00456

Barnes, C. M., Watkins, T., \& Klotz, A. (2020). An exploration of employee dreams: The dream-based overnight carryover of emotional experiences at work. Sleep Health. https://doi.org/10.1016/j.sleh.2020.11.001

Blagrove, M., Farmer, L., \& Williams, E. (2004). The relationship of nightmare frequency and nightmare distress to well-being. Journal of Sleep Research, 13(2), 129-136. https://doi.org/10.1111/j.1365-2869.2004.00394.x

Bulkeley, K., \& Graves, M. (2018). Using the LIWC program to study dreams. Dreaming, 28(1), 43-58. https://doi.org/10.1037/drm0000071

Carr, M., Haar, A., Amores, J., Lopes, P., Bernal, G., Vega, T., Rosello, O., Jain, A., \& Maes, P. (2020). Dream engineering: Simulating worlds through sensory stimulation. Consciousness and Cognition, 83, 102955. https://doi.org/10.1016/j.concog.2020.102955

Carr, M., Summers, R., Bradshaw, C., Newton, C., Ellis, L., Johnston, E., \& Blagrove, M. (2020). Frontal Brain Activity and Subjective Arousal During Emotional Picture Viewing in Nightmare Sufferers. Frontiers in Neuroscience, 14. https://doi.org/10.3389/fnins.2020.585574

Fireman, G. D., Levin, R., \& Pope, A. W. (2014). Narrative qualities of bad dreams and nightmares. Dreaming, 24(2), 112-124. https://doi.org/10.1037/a0035791

Hawkins II, R. C., \& Boyd, R. L. (2017). Such stuff as dreams are made on: Dream language, LIWC norms, and personality correlates. Dreaming, 27(2), 102-121. https://doi.org/10.1037/drm0000049

Konkoly, K., \& Burke, C. T. (2019). Can learning to lucid dream promote personal growth? Dreaming, 29(2), 113-126. https://doi.org/10.1037/drm0000101

Köthe, M., \& Pietrowsky, R. (2001). Behavioral Effects of Nightmares and Their Correlations to Personality Patterns. Dreaming, 11(1), 43-52. https://doi.org/10.1023/A:1009468517557

Lee, M.-N., \& Kuiken, D. (2015). Continuity of reflective awareness across waking and dreaming states. Dreaming, 25(2), 141-159. https://doi.org/10.1037/a0039147

Nielsen, T. A., Zadra, A. L., Simard, V., Saucier, S., Stenstrom, P., Smith, C., \& Kuiken, D. (2003). The Typical Dreams of Canadian University Students. Dreaming, 13(4), 211235. https://doi.org/10.1023/B:DREM.0000003144.40929.0b

Paquet, C., Cogan, C. M., \& Davis, J. L. (2020). A quantitative text analysis approach to describing posttrauma nightmares in a treatment-seeking population. Dreaming, 30(1), 54-67. https://doi.org/10.1037/drm0000128

Pennebaker, J. W., Boyd, R. L., Jordan, K., \& Blackburn, K. (2015). The Development and Psychometric Properties of LIWC2015. https://repositories.lib.utexas.edu/handle/2152/31333

Robert, G., \& Zadra, A. (2014). Thematic and Content Analysis of Idiopathic Nightmares and Bad Dreams. Sleep, 37(2), 409-417. https://doi.org/10.5665/sleep.3426

Schredl, M. (2000). The effect of dreams on waking life. Sleep and Hypnosis, 2(3), 120-124. Schredl, M. (2009). Effect of dreams on daytime mood: The effects of gender and personality. Sleep and Hypnosis, 11(2), 51-57. 
DREAMS AND MORNING MOOD

268 Schredl, M., \& Göritz, A. S. (2018). Nightmare Themes: An Online Study of Most Recent Nightmares and Childhood Nightmares. Journal of Clinical Sleep Medicine, 14(03), 465-471. https://doi.org/10.5664/jcsm.7002

Schredl, M., \& Reinhard, I. (2010). The Continuity between Waking Mood and Dream Emotions: Direct and Second-Order Effects. Imagination, Cognition and Personality, 29(3), 271-282. https://doi.org/10.2190/lC.29.3.f

Stocks, A., Carr, M., Mallett, R., Konkoly, K., Hicks, A., Crawford, M., Schredl, M., \& Bradshaw, C. (2020). Dream lucidity is associated with positive waking mood. Consciousness and Cognition, 83, 102971. https://doi.org/10.1016/j.concog.2020.102971

Stoet, G. (2017). PsyToolkit: A Novel Web-Based Method for Running Online Questionnaires and Reaction-Time Experiments. Teaching of Psychology, 44(1), 24-31. https://doi.org/10.1177/0098628316677643

Vallat, R. (2018). Pingouin: Statistics in Python. Journal of Open Source Software, 3(31), 1026. https://doi.org/10.21105/joss.01026

Vandekerckhove, M., \& Cluydts, R. (2010). The emotional brain and sleep: An intimate relationship. Sleep Medicine Reviews, 14(4), 219-226. https://doi.org/10.1016/j.smrv.2010.01.002

Walker, M. P., \& van der Helm, E. (2009). Overnight therapy? The role of sleep in emotional brain processing. Psychological Bulletin, 135(5), 731-748. https://doi.org/10.1037/a0016570

Yu, C. K.-C. (2007). Emotions before, during, and after dreaming sleep. Dreaming, 17(2), 7386. https://doi.org/10.1037/1053-0797.17.2.73 
Supplementary Information for

\title{
The relationship between dreams and subsequent morning mood using self-reports and text analysis
}

\author{
Mallett, Picard-Deland, Pigeon, Wary, Grewal, Blagrove, \& Carr
}

\section{Contents}

S1. Table of descriptive statistics for summary variables.

S2. Table of LIWC results controlling for sleep efficiency. 
S1. Table of descriptive statistics for summary variables. Values are averaged withinparticipant, across mornings, before averaging across all participants.

\begin{tabular}{|c|c|c|c|c|c|}
\hline & mean & std & median & $\min$ & $\max$ \\
\hline number of dream reports in study & 14.59 & 9.39 & 12 & 2 & 38 \\
\hline sleep efficiency & 93.39 & 3.97 & 93.51 & 77.93 & 99.22 \\
\hline dream word count & 76.59 & 32.82 & 71.17 & 32.50 & 160.27 \\
\hline dream negative emotion & 4.38 & 1.74 & 4 & 1.50 & 8.75 \\
\hline dream positive emotion & 3.00 & 1.22 & 2.86 & 1 & 5.69 \\
\hline wake negative emotion & 3.04 & 1.58 & 2.82 & 1 & 8.12 \\
\hline wake positive emotion & 3.22 & 1.39 & 2.85 & 1 & 6.42 \\
\hline positive emotion LIWC & 1.83 & 1.13 & 1.61 & 0 & 5.72 \\
\hline anxiety LIWC & 0.71 & 0.42 & 0.70 & 0 & 1.54 \\
\hline anger LIWC & 0.67 & 0.51 & 0.58 & 0 & 2.74 \\
\hline sad LIWC & 0.34 & 0.34 & 0.30 & 0 & 1.50 \\
\hline work LIWC & 1.72 & 0.92 & 1.55 & 0 & 4.40 \\
\hline leisure LIWC & 2.22 & 0.94 & 2.01 & 0.55 & 5.19 \\
\hline home LIWC & 1.23 & 0.71 & 1.16 & 0 & 3.15 \\
\hline money LIWC & 0.41 & 0.39 & 0.38 & 0 & 1.55 \\
\hline religion LIWC & 0.10 & 0.16 & 0.03 & 0 & 0.83 \\
\hline death LIWC & 0.32 & 0.37 & 0.18 & 0 & 1.69 \\
\hline see LIWC & 1.14 & 0.60 & 1.09 & 0 & 3.05 \\
\hline hear LIWC & 0.46 & 0.36 & 0.40 & 0 & 1.62 \\
\hline feel LIWC & 0.88 & 0.72 & 0.76 & 0 & 3.62 \\
\hline body LIWC & 0.75 & 0.84 & 0.45 & 0 & 4.24 \\
\hline health LIWC & 0.61 & 0.54 & 0.51 & 0 & 2.11 \\
\hline sexual LIWC & 0.07 & 0.17 & 0 & 0 & 0.86 \\
\hline ingestion LIWC & 0.60 & 0.55 & 0.45 & 0 & 2.58 \\
\hline family LIWC & 0.88 & 0.65 & 0.75 & 0 & 2.34 \\
\hline friend LIWC & 0.83 & 0.58 & 0.87 & 0 & 1.99 \\
\hline male LIWC & 1.40 & 1.17 & 1.25 & 0 & 4.42 \\
\hline female LIWC & 1.01 & 0.73 & 1.06 & 0 & 3.41 \\
\hline i LIWC & 8.61 & 2.11 & 8.23 & 4.66 & 15.42 \\
\hline we LIWC & 1.20 & 1.06 & 0.98 & 0 & 4.32 \\
\hline affiliation LIWC & 3.17 & 1.83 & 3.16 & 0 & 7.53 \\
\hline achievement LIWC & 1.43 & 0.53 & 1.43 & 0 & 2.72 \\
\hline power LIWC & 2.26 & 0.80 & 2.23 & 0 & 4.06 \\
\hline reward LIWC & 1.20 & 0.61 & 1.12 & 0 & 2.66 \\
\hline risk LIWC & 0.76 & 1.12 & 0.51 & 0 & 7.64 \\
\hline
\end{tabular}


S2. Table of LIWC results controlling for sleep efficiency. Note that due to some unanswered sleep quality questions, this analysis has 24 less morning reports included.

\begin{tabular}{|c|c|c|c|c|}
\hline \multirow[b]{2}{*}{ LIWC category } & \multicolumn{2}{|r|}{ LIWC } & \multicolumn{2}{|c|}{ sleep efficiency } \\
\hline & ß $(95 \% \mathrm{Cl})$ & $p$ & B (95\% Cl) & $p$ \\
\hline anxiety & $.14(.07, .20)$ & $<.001$ & $-.04(-.11, .03)$ & .230 \\
\hline anger & $.06(-.01, .12)$ & .07 & $-.04(-.11, .03)$ & .237 \\
\hline sadness & $.03(-.03, .10)$ & .307 & $-.04(-.11, .03)$ & .213 \\
\hline pos emo & $-.16(-.23,-.09)$ & $<.001$ & $-.04(-.11, .03)$ & .257 \\
\hline death & $.09(.03, .16)$ & .005 & $-.05(-.11, .02)$ & .189 \\
\hline religion & $-.01(-.07, .05)$ & .703 & $-.04(-.11, .03)$ & .231 \\
\hline home & $-.02(-.08, .04)$ & .540 & $-.04(-.11, .03)$ & .231 \\
\hline work & $-.02(-.08, .04)$ & .541 & $-.04(-.11, .03)$ & .239 \\
\hline money & $-.07(-.14,-.01)$ & .036 & $-.04(-.11, .03)$ & .243 \\
\hline leisure & $-.10(-.17,-.04)$ & .002 & $-.04(-.11, .03)$ & .232 \\
\hline$i$ & $.12(.06, .19)$ & $<.001$ & $-.04(-.11, .03)$ & .244 \\
\hline male & $.08(.007, .143)$ & .030 & $-.04(-.11, .03)$ & .267 \\
\hline female & $.05(-.01, .12)$ & .125 & $-.04(-.11, .03)$ & .248 \\
\hline friend & $.03(-.04, .09)$ & .407 & $-.04(-.11, .03)$ & .241 \\
\hline family & $-.02(-.08, .04)$ & .478 & $-.04(-.11, .03)$ & .233 \\
\hline we & $-.09(-.16,-.02)$ & .013 & $-.04(-.11, .03)$ & .275 \\
\hline risk & $.06(-.00, .13)$ & .060 & $-.05(-.12, .02)$ & .191 \\
\hline power & $.01(-.05, .08)$ & .700 & $-.04(-.11, .03)$ & .239 \\
\hline achieve & $-.02(-.08, .04)$ & .525 & $-.04(-.11, .03)$ & .238 \\
\hline reward & $-.05(-.11, .01)$ & .129 & $-.04(-.11, .03)$ & .211 \\
\hline affiliation & $-.06(-.13, .01)$ & .100 & $-.04(-.11, .03)$ & .251 \\
\hline body & $.07(-.00, .13)$ & .057 & $-.03(-.10, .04)$ & .350 \\
\hline feel & $.05(-.02, .12)$ & .158 & $-.04(-.11, .03)$ & .211 \\
\hline hear & $.03(-.03, .10)$ & .319 & $-.04(-.11, .03)$ & .260 \\
\hline health & $.02(-.05, .08)$ & .629 & $-.04(-.11, .03)$ & .232 \\
\hline see & $.01(-.05, .07)$ & .739 & $-.04(-.11, .03)$ & .245 \\
\hline sexual & $-.04(-.10, .03)$ & .228 & $-.04(-.11, .03)$ & .264 \\
\hline ingestion & $-.09(-.15,-.02)$ & .008 & $-.05(-.12, .02)$ & .182 \\
\hline
\end{tabular}

